\title{
Atividade biológica in vitro de própolis e óleos essenciais sobre o fungo Colletotrichum musae isolado de bananeira (Musa spp.)
}

BARBOSA, M.S.'; VIEIRA, G.H.C..,*; TEIXEIRA, A.V. ${ }^{3}$

${ }^{1,3}$ Alunos do Curso de Agronomia, Bolsistas de Iniciação Científica FUNDECT/CNPq/UEMS, Universidade Estadual de Mato Grosso do Sul (UEMS), Unidade Universitária de Cassilândia, Rodovia MS 306, km 6,4, Cassilândia/MS-Brasil, CEP: 79540-000; '2Professor Doutor do Curso de Agronomia, Universidade Estadual de Mato Grosso do Sul (UEMS), Unidade Universitária de Cassilândia, Rodovia MS 306, km 6,4, Cassilândia/ MS-Brasil, CEP: 79540-000, *gcv@uems.br.

RESUMO: No Brasil existem várias doenças fúngicas que acometem a bananeira. Destas, pode-se citar a antracnose, responsável por grandes prejuízos à cultura, cujo agente causal é o fungo Colletotrichum musae. A principal forma de controle dessa enfermidade é através da aplicação de fungicidas a base de tiabendazol ou tiofanato metílico. Esse manejo, embora eficiente, favorece o desenvolvimento de resistência do patógeno, causa danos ao ambiente e ao produtor, deixando ainda resíduos nos frutos. Esses fatores têm favorecido a busca por substâncias alternativas com capacidade de controlar o fungo e que não sejam nocivas ao ambiente e, principalmente, que sejam seguras ao consumidor final. Dentre as opções, surge o interesse pelo uso de certos óleos essenciais e da própolis, ambos conhecidos por possuírem propriedades fungicidas. O presente trabalho foi desenvolvido com o objetivo de determinar o potencial fungitóxico "in vitro" da própolis e dos óleos essenciais de palmarosa (Cymbopogon martinii), de teatree (Melaleuca alternifolia), de cravo (Eugenia caryophyllata), e de eucalipto (Corymbia citriodora), sobre Colletotrichum musae. O desenvolvimento experimental consistiu em adicionar inóculos fúngicos de $5 \mathrm{~mm}$, obtidos a partir de colônias puras, ao meio de cultura BDA (batata-dextrose-ágar) acrescido das referidas substâncias em diferentes concentrações ( 0 , $25,50,75,100$ e $125 \mu \mathrm{L} / \mathrm{L}$ ). Paralelo aos tratamentos realizou-se teste com o fungicida padrão para comparações das médias. A eficiência das substâncias sobre o fungo foi determinada através das avaliações do crescimento micelial das colônias (média de duas medidas diametralmente opostas). Os valores de crescimento micelial obtidos foram utilizados também para o cálculo do índice de velocidade de crescimento micelial. O delineamento experimental utilizado foi inteiramente casualizado em esquema fatorial $5 \times 6+1$, (cinco substâncias em seis concentrações + fungicida), com cinco repetições. Os óleos de tea tree, cravo e palmarosa foram eficientes no controle do fungo Colletotrichum musae não diferindo do fungicida a partir da dose de $50 \mu \mathrm{L} / \mathrm{L}$ em todas as avaliações, apresentando potencial para controle em cultivos orgânicos ou em sistemas de manejo integrado.

Palavras-chave: Produção orgânica, Fungos, Desenvolvimento sustentável, Própolis.

ABSTRACT: In vitro biological activity of propolis and essential oils on the fungus Colletotrichum musae isolated from banana Musa spp. In Brazil there are several fungi that cause diseases on banana plants. These include the "anthracnose", which is responsible for major crop losses and whose causative agent is the fungus Colletotrichum musae. The main way to control this disease is through the application of fungicides based on thiabendazole and thiophanate-methyl. Although this management is effective, it favors the development of pathogen resistance, which causes damage to the environment and producer and also leaves residues in fruits. These factors have encouraged the search for alternative substances to control the fungus and that are not harmful to the environment and particularly to the final consumer. Among the options, there is interest in the use of essential oils and propolis, both known to have antifungal activity. The present work was developed with the objective of determining the potential of propolis and essential oils of palmarosa (Cymbopogon martinii), tea tree (Melaleuca alternifolia), clove (Eugenia caryophyllata) and eucalyptus (Corymbia citriodora) in the in vitro 
control of the fungus Colletotrichum musae. The experimental development consisted in adding 5 $\mathrm{mm}$ fungal inoculants, obtained from pure colonies, in PDA culture (potato-dextrose-agar) plus the aforementioned substances in different concentrations $(0,25,50,75,100$ and $125 \mu \mathrm{L} / \mathrm{L})$. At the same time as these treatments, we carried out a test with the fungicide to compare the averages. The efficiency of the substances on the fungus was determined through evaluations of the mycelial growth of the colonies (average of two diametrically opposed measures). The values of mycelial growth obtained were also used for the calculation of the speed index of the mycelial growth. The experimental design was completely randomized in $5 \times 6+1$ ( 5 substances in 6 concentrations + fungicide) factorial design, with 5 repetitions. The tea tree, clove and palmarosa oils were efficient in the control of the fungus Colletotrichum musae, which can be used as a control option in organic crops or in integrated management systems.

Key words: Organic production, Essential oils, Fungi, Sustainable development, Propolis.

\section{INTRODUÇÃO}

No Brasil existem várias doenças fúngicas que afetam a bananeira. Dentre as principais, pode-se citar a antracnose, causada pelo fungo Colletotrichum musae, responsável por grandes prejuízos à cultura (Coelho et al., 2010). O controle dessa enfermidade é feito por imersão ou pulverização dos frutos em fungicidas a base de tiabendazol e imazalil, em concentrações que variam de 200 a 400 ppm (Brasil, 2010).

Essa patogenia se desenvolve tanto no período pré, como pós-colheita. Na pré-colheita, o fruto é contaminado pelo patógeno que permanece quiescente até o início da maturação (Goos \& Tschirsch, 1962), provavelmente em decorrência do tanino presente nos frutos verdes (Cordeiro \& Kimati, 1997). No período pós-colheita, quando os frutos encontram-se em fase de maturação, as infecções quiescentes se manifestam juntamente com infecções secundárias, chamadas de infecções não quiescentes (Ploetz et al., 2003). Em estágio avançado a doença pode apresentar ainda lesões agrupadas, responsáveis pelo descarte do produto (Cordeiro \& Matos, 2005; Sponholz et al., 2004).

As estratégias de manejo para o controle do fungo compreendem modernos sistemas de embalagem e transporte, medidas de controle a campo e principalmente o controle químico. Este último é responsável por causar danos ao ambiente e a saúde humana, além de favorecer o surgimento de estirpes resistentes (Cruz et al., 2010).

A necessidade de minimizar os impactos causados por agrotóxicos, associadaa procura crescente por produtos orgânicos, tem favorecido o desenvolvimento de estudos com substâncias alternativas que possam substituir os produtos químicos usados no controle desses microrganismos.

Dentre os produtos naturais usados com esse objetivo, encontram-se a própolis e os óleos essenciais. A própolis devido às suas propriedades antimicrobianas, antifúngicas, antioxidantes, antiviral e antiprotozoárias vêm sendo amplamente usada no controle de microorganismos patogênicos (Pereira et al., 2002; Melo et al., 2009).

Assim como a própolis, alguns óleos essenciais caracterizam-se por apresentarem ações bactericida e fungicida, além de atuarem contra herbívoros na proteção de plantas na natureza (Bakkali et al., 2008), sendo uma opção viável no controle alternativo de patógenos que acometem as plantas (Stangarlin, 2007).

Considerando a importância da banana como fonte alimentar e principalmente a necessidade de garantir ao consumidor um produto isento de agrotóxicos, o presente trabalho foi desenvolvido com o objetivo de determinar o potencial "in vitro" da própolis e de diferentes óleos essenciais no controle do fungo Colletotrichum musae (Arx), causador da antracnose na bananeira.

\section{MATERIAL E MÉTODOS}

O trabalho foi conduzido no laboratório de Fitossanidade da Universidade Estadual do Mato Grosso do Sul, município de Cassilândia/MS (19॰ 06'48" S; 51॰44'03" W), de agosto/2012 a julho/2013.

\section{Produção da própolis}

A própolis foi produzida com auxílio de coletores de própolis inteligente (CPI), instalados em colméias de Apis mellifera. Os coletores foram instalados em colônias com dois anos de formação, produzidas a partir de divisão de enxames populosos. Todas as colméias do apiário são submetidas à substituição anual de rainha. Essas colméias foram mantidas em área de cerrado (savana arbórea aberta), cuja flora apícola em floração durante o período de coleta da própolis, que correspondeu aos meses de abril a julho, é predominantemente representada pelas espécies Duguetia furfuraceae (St. Hil.) Benth \& Hook, Eupatorium sp., Mikania sp., Vernonia sp., Pyrostegia venusta (Ker-Gawl.) Miers, Banisteriopsis sp. E Solanum lycocarpum A. St-Hil.

Rev. Bras. PI. Med., Campinas, v.17, n.2, p.254-261, 2015. 
Após coletada a própolis foi imediatamente imersa em álcool de cereais na concentração de $30 \%$, conforme preconiza a literatura (Silva, 2003), sendo mantida em recipiente estéril ao abrigo da luz até o início dos testes. Essa solução foi submetida a novas diluições em água destilada, seguindo-se as mesmas concentrações usadas nos testes com os óleos essenciais.

\section{Óleos essenciais}

Foram testados os óleos de palmarosa (Cymbopogon Martinii Roxb. Wats. var. motia Burk.), tea tree (Melaleuca alternifolia Cheel.), cravo (Eugenia caryophyllata Thunb.) e eucalipto (Corymbia citriodora Hook.). Os óleos essenciais foram adquiridos de uma empresa especializada, registrada na Anvisa sob o $n^{\circ}$ CEVS 354340218477-000401-1-8.

\section{Isolamento do fungo}

$O$ fungo foi isolado diretamente de frutos com sintomas e inoculados em meio de cultura BDA (batata-dextrose-ágar) acondicionado em placas de Petri de $8,8 \mathrm{~cm}$, mantidas em uma câmara climática com temperatura de $27^{\circ} \mathrm{C}$ e fotoperíodo de 12 horas.

Todos os procedimentos foram realizados em ambiente asséptico (câmara de fluxo laminar). As colônias puras foram utilizadas posteriormente para a realização dos tratamentos com própolis e óleos essenciais.

\section{Determinação da atividade antifúngica da própolis e óleos essenciais}

A atividade antifúngica da própolis e dos óleos essenciais foi determinada através do desenvolvimento micelial do fungo em meio de cultura acrescido das referidas substâncias em diferentes dosagens. Para tanto, discos da colônia fúngica com $5 \mathrm{~mm}$ de diâmetro foram transferidos para meios BDA (batata-dextrose-ágar) acondicionados em placas de Petri. A própolis e os óleos essenciais de palmarosa (Cymbopogon martinii), "tea tree" (Melaleuca alternifolia), cravo (Eugenia caryophyllata) e eucalipto (Corymbia citriodora), foram adicionados aos meios BDA ainda fundente, nas seguintes concentrações: 1) $0 \mu \mathrm{L} / \mathrm{L}$ (testemunha), 2) $25 \mu \mathrm{L} / \mathrm{L}$, 3) $50 \mu \mathrm{L} / \mathrm{L}$, 4) $75 \mu \mathrm{L} / \mathrm{L}$, 5) $100 \mu \mathrm{L} / \mathrm{L}$ e 6) $125 \mu \mathrm{L} / \mathrm{L}$. Paralelo aos testes com as substâncias realizou-se um tratamento com o fungicida Tecto SC (tiabendazol) na dose de $25 \mu \mathrm{L} / \mathrm{L}$, sendo os resultados usados para comparações. Após a inoculação dos fungos as placas foram mantidas em BOD a $27^{\circ} \mathrm{C}$, com fotoperíodo de 12 horas.

As avaliações foram realizadas através de medições do diâmetro das colônias (média de duas medidas diametralmente opostas), com auxilio de um paquímetro, durante seis períodos $48,72,96$, 120, 144 e 168 horas após a repicagem.

Os valores de crescimento micelial obtidos foram utilizados também para o cálculo do IVCM (índice de velocidade de crescimento micelial), conforme Oliveira (1991). Este índice foi determinado através da equação: $\Sigma(D-D a) / N$, sendo $D=$ diâmetro médio atual da colônia, $\mathrm{Da}=$ diâmetro médio da colônia do dia anterior, $\mathrm{N}=$ número de dias após a inoculação.

O delineamento experimental utilizado foi inteiramente casualizado, em esquema fatorial 5 x $6+1$ (cinco substâncias em seis concentrações + fungicida), com cinco repetições. As variáveis foram submetidas à análise de variância e as médias comparadas pelo teste Scott-Knott a 5\% de probabilidade. Os resultados obtidos no último período de avaliação foram submetidos à análise de regressão. As análises estatísticas foram realizadas pelo programa computacional SISVAR (Ferreira, 2011).

\section{RESULTADOS E DISCUSSÃO}

O efeito inibitório dos óleos essenciais sobre Colletotrichum musae estão dispostos na Tabela 1.

Os melhores resultados "in vitro" para a inibição do crescimento micelial do fungo $C$. musae foram obtidos pelos óleos essenciais de cravo e palmarosa, seguidos por tea tree (Tabela 1 e Figura 1). Para os óleos de cravo e palmarosa, os resultados observados não diferiram estatisticamente dos resultados obtidos com o fungicida desde a menor dose testada, que correspondeu a $25 \mu \mathrm{L} / \mathrm{L}$, em todos os períodos avaliados (Tabela 1). Para o óleo de tea tree, os resultados obtidos para as concentrações acima de $50 \mu \mathrm{L} / \mathrm{L}$ também não diferiram do fungicida, sugerindo que essas substâncias, nas respectivas doses, apresentam ação fungicida sobre o fungo C. musae.

O óleo de eucalipto e a própolis apresentaram baixa influência sobre o fungo C. musae. Os dados apresentados na Tabela 1 para essas duas substâncias indicam que até a terceira avaliação, a inibição do fungo foi dose dependente, ou seja, observa-se uma diminuição no tamanho da colônia fúngica com o aumento da dose da substância testada, chegando, no caso do eucalipto a não diferir estatisticamente dos resultados obtidos com o fungicida no período de 48 horas da repicagem. No entanto, a partir da terceira avaliação para a própolis e quinta para o eucalipto essa relação não foi mais observada.

Os resultados obtidos neste estudo para o óleo de eucalipto foram semelhantes aos

Rev. Bras. PI. Med., Campinas, v.17, n.2, p.254-261, 2015. 
TABELA 1. Crescimento micelial do fungo Colletotrichum musae na presença de cinco substâncias alternativas em diferentes concentrações. Cassilândia/MS, 2013.

\begin{tabular}{|c|c|c|c|c|c|}
\hline & & rescimento & m) & & \\
\hline Dosagens & & & avaliação & & \\
\hline$(\mu \mathrm{L} / \mathrm{L})$ & Própolis & Eucalipto & Tea tree & Cravo & Palmarosa \\
\hline Fungicida & $0,00 \mathrm{aD}$ & $0,00 \mathrm{aE}$ & $0,00 \mathrm{aB}$ & $0,00 \mathrm{Ab}$ & $0,00 \mathrm{aB}$ \\
\hline Testemunha & $2,54 \mathrm{aA}$ & $2,54 \mathrm{aA}$ & $2,54 \mathrm{aA}$ & $2,54 \mathrm{aA}$ & $2,54 \mathrm{aA}$ \\
\hline 25 & $1,36 \mathrm{aB}$ & $1,09 \mathrm{bB}$ & $0,00 \mathrm{cB}$ & $0,00 \mathrm{cB}$ & $0,00 \mathrm{cB}$ \\
\hline 50 & $1,32 \mathrm{aB}$ & $0,86 \mathrm{bC}$ & $0,00 \mathrm{cB}$ & $0,02 \mathrm{cB}$ & $0,00 \mathrm{cB}$ \\
\hline 75 & $1,14 \mathrm{aC}$ & $0,40 \mathrm{bD}$ & $0,00 \mathrm{cB}$ & $0,02 \mathrm{cB}$ & $0,00 \mathrm{cB}$ \\
\hline 100 & $1,06 \mathrm{aC}$ & $0,04 \mathrm{bE}$ & $0,00 \mathrm{bB}$ & $0,04 \mathrm{bB}$ & $0,00 \mathrm{bB}$ \\
\hline 125 & $1,06 \mathrm{aC}$ & $0,00 \mathrm{bE}$ & $0,00 \mathrm{bB}$ & $0,00 \mathrm{bB}$ & $0,00 \mathrm{bB}$ \\
\hline & & & avaliação & & \\
\hline Fungicida & $0,00 \mathrm{aF}$ & $0,00 \mathrm{aG}$ & $0,00 \mathrm{aB}$ & $0,00 \mathrm{aB}$ & $0,00 a B$ \\
\hline Testemunha & $4,90 a A$ & $4,90 a A$ & $4,90 a A$ & $4,90 a A$ & $4,90 a A$ \\
\hline 25 & $3,21 \mathrm{aB}$ & $3,04 a B$ & $0,16 \mathrm{bB}$ & $0,02 \mathrm{bB}$ & $0,00 \mathrm{bB}$ \\
\hline 50 & $2,82 a C$ & $2,74 a C$ & $0,00 \mathrm{bB}$ & $0,04 \mathrm{bB}$ & $0,00 \mathrm{bB}$ \\
\hline 75 & $2,59 a D$ & $2,08 b D$ & $0,00 \mathrm{cB}$ & $0,04 c B$ & $0,00 \mathrm{cB}$ \\
\hline 100 & $2,36 \mathrm{aE}$ & $1,15 \mathrm{bE}$ & $0,00 \mathrm{aB}$ & $0,04 a B$ & $0,00 a B$ \\
\hline 125 & $2,34 \mathrm{aE}$ & $0,24 \mathrm{bF}$ & $0,00 \mathrm{cB}$ & $0,00 \mathrm{cB}$ & $0,00 \mathrm{cB}$ \\
\hline & & & valiação & & \\
\hline Fungicida & $0,00 \mathrm{aE}$ & $0,00 \mathrm{aG}$ & $0,00 \mathrm{aC}$ & $0,00 \mathrm{aB}$ & $0,00 \mathrm{aB}$ \\
\hline Testemunha & $7,30 a A$ & $7,30 a A$ & $7,30 a A$ & $7,30 a A$ & $7,30 a A$ \\
\hline 25 & $5,10 \mathrm{aB}$ & $4,73 \mathrm{bB}$ & $0,44 \mathrm{cB}$ & $0,02 d B$ & $0,00 \mathrm{~dB}$ \\
\hline 50 & $4,22 \mathrm{aC}$ & $4,45 \mathrm{aC}$ & $0,00 \mathrm{bC}$ & $0,04 \mathrm{bB}$ & $0,00 \mathrm{bB}$ \\
\hline 75 & $4,06 a C$ & $3,64 b D$ & $0,00 \mathrm{cC}$ & $0,04 \mathrm{cB}$ & $0,00 \mathrm{cB}$ \\
\hline 100 & $3,67 \mathrm{aD}$ & $2,42 \mathrm{bE}$ & $0,00 \mathrm{cC}$ & $0,04 c B$ & $0,00 \mathrm{cB}$ \\
\hline 125 & $3,64 a \mathrm{D}$ & $1,20 \mathrm{bF}$ & $0,00 \mathrm{cC}$ & $0,00 \mathrm{cB}$ & $0,00 \mathrm{cB}$ \\
\hline & & & aliação (1 & & \\
\hline Fungicida & $0,00 \mathrm{aE}$ & $0,00 \mathrm{aF}$ & $0,00 \mathrm{aC}$ & $0,00 \mathrm{aB}$ & $0,00 a B$ \\
\hline Testemunha & $8,30 a A$ & $8,30 a A$ & $8,30 a A$ & $8,30 a A$ & $8,30 a A$ \\
\hline 25 & $6,77 a \mathrm{~B}$ & $6,51 \mathrm{aB}$ & $0,73 b B$ & $0,02 \mathrm{cB}$ & $0,00 \mathrm{cB}$ \\
\hline 50 & $5,54 \mathrm{bC}$ & $6,22 \mathrm{aB}$ & $0,00 \mathrm{cC}$ & $0,04 \mathrm{cB}$ & $0,00 \mathrm{cB}$ \\
\hline 75 & $5,36 a C$ & $5,22 \mathrm{aC}$ & $0,00 \mathrm{bC}$ & $0,04 \mathrm{bB}$ & $0,00 \mathrm{bB}$ \\
\hline 100 & $4,69 \mathrm{aD}$ & $3,95 b D$ & $0,00 c C$ & $0,04 \mathrm{cB}$ & $0,00 \mathrm{cB}$ \\
\hline 125 & $4,95 \mathrm{aD}$ & $2,47 \mathrm{bE}$ & $0,00 c C$ & $0,00 \mathrm{cB}$ & $0,00 \mathrm{cB}$ \\
\hline & & & liação (14 & & \\
\hline Fungicida & $0,00 \mathrm{aE}$ & $0,00 \mathrm{aF}$ & $0,00 \mathrm{aC}$ & $0,00 \mathrm{aB}$ & $0,00 \mathrm{aB}$ \\
\hline Testemunha & $8,30 a A$ & $8,30 a A$ & $8,30 a A$ & $8,30 \mathrm{aA}$ & $8,30 a A$ \\
\hline 25 & $8,30 a A$ & $7,93 a A$ & $1,28 \mathrm{bB}$ & $0,02 \mathrm{cB}$ & $0,00 \mathrm{cB}$ \\
\hline 50 & $6,39 \mathrm{bB}$ & $7,45 \mathrm{aB}$ & $0,00 \mathrm{cC}$ & $0,04 \mathrm{cB}$ & $0,00 \mathrm{cB}$ \\
\hline 75 & $6,50 \mathrm{bB}$ & $7,00 \mathrm{aC}$ & $0,00 c C$ & $0,04 \mathrm{cB}$ & $0,00 \mathrm{cB}$ \\
\hline 100 & $5,39 a D$ & $5,32 \mathrm{aD}$ & $0,00 \mathrm{bC}$ & $0,04 \mathrm{bB}$ & $0,00 \mathrm{bB}$ \\
\hline 125 & $5,97 \mathrm{aC}$ & $4,00 \mathrm{bE}$ & $0,00 \mathrm{cC}$ & $0,00 \mathrm{cB}$ & $0,00 \mathrm{cB}$ \\
\hline & & & iação (16 & & \\
\hline Fungicida & $0,00 \mathrm{aF}$ & $0,00 \mathrm{aE}$ & $0,00 \mathrm{aC}$ & $0,00 a B$ & $0,00 a B$ \\
\hline Testemunha & $8,30 a A$ & $8,30 a A$ & $8,30 a A$ & $8,30 a A$ & $8,30 a A$ \\
\hline 25 & $8,30 a A$ & $8,30 a A$ & $1,96 \mathrm{bB}$ & $0,02 \mathrm{cB}$ & $0,00 \mathrm{cB}$ \\
\hline 50 & $6,68 b C$ & $8,30 a A$ & $0,03 c C$ & $0,04 \mathrm{cB}$ & $0,00 \mathrm{cB}$ \\
\hline 75 & $7,21 \mathrm{aB}$ & $7,69 a \mathrm{a}$ & $0,00 \mathrm{cC}$ & $0,04 \mathrm{cB}$ & $0,00 \mathrm{cB}$ \\
\hline 100 & $5,57 \mathrm{bE}$ & $6,15 \mathrm{aC}$ & $0,00 \mathrm{cC}$ & $0,04 \mathrm{cB}$ & $0,00 \mathrm{cB}$ \\
\hline 125 & $6,14 a \mathrm{D}$ & $5,42 \mathrm{bD}$ & $0,00 \mathrm{cC}$ & $0,00 \mathrm{cB}$ & $0,00 \mathrm{cB}$ \\
\hline
\end{tabular}

Letras iguais minúsculas nas linhas e maiúsculas nas colunas não diferem significativamente no teste Scott-Knott a 5\%. Testes com o fungicida foram realizados separadamente sendo apresentado apenas para comparações. 
observados por Salgado et al. (2003), em estudos realizados com óleos extraídos de diferentes espécies de eucalipto. Esses autores observaram que a taxa de inibição do crescimento micelial do fungo é proporcional ao aumento da dose testada.

Quando comparado à eficiência das dosagens entre as substâncias, os óleos de cravo e palmarosa, seguidos por tea tree também apresentaram os melhores resultados para a inibição do crescimento micelial do fungo. Os dois primeiros não diferiram significativamente entre si e nem dos resultados obtidos para o fungicida desde a menor concentração testada ( $25 \mu \mathrm{L} / \mathrm{L})$, em todas as avaliações realizadas. Para tea tree, os valores observados a partir da concentração de 50 $\mu \mathrm{L} / \mathrm{L}$ também não diferiram do fungicida e nem dos óleos de cravo e palmarosa. A própolis e o eucalipto diferiram entre si e dos demais tratamentos, sendo as substâncias que apresentaram as menores eficiências no controle do fungo.

Os níveis de eficiência do óleo de eucalipto e da própolis foram diminuindo a partir da avaliação realizada após $120 \mathrm{~h}$ da inoculação, sendo ainda menores nas avaliações subseqüentes. Nota-se que nesse período a própolis nas dosagens de 50 e $75 \mu \mathrm{L} / \mathrm{L}$ e nas dosagens de 100 e $125 \mu \mathrm{L} / \mathrm{L}$ não diferiram entre $\mathrm{si}$, sendo os maiores valores de crescimento micelial observados dentre todas as substâncias testadas.

Embora muitos estudos relatem a eficiência da própolis e de óleos essenciais no controle de fungos e outros microorganismos (Fernandes et al., 2007; Probst et al., 2011; Monzote et al., 2012), há casos em que essa eficiência não é confirmada. Marini et al. (2012), estudaram a eficiência in vitro do extrato etanólico de própolis sobre os fungos Phakopsora euvitis, Pseudocercospora vitis e Elsinoe ampelina, obtendo resultados pouco expressivos.

No entanto, Longhini et al. (2007) determinaram a eficiência do extrato de própolis sobre leveduras isoladas de onicomicoses, observando que essa substância mesmo em pequenas concentrações possui ação fungicida sobre esse patógeno. Fernandes et al. (2007) relataram que o extrato etanólico de própolis na concentração de $0,2 \mathrm{mg}^{\mathrm{mL}} \mathrm{mL}^{-1}$ é eficiente no controle do fungo Cryptococcus neoformans.

A divergência observada em alguns resultados obtidos com a própolis deve-se em parte a sua origem. Sabe-se que essa substância possui uma complexa composição química, que está diretamente relacionada a flora fornecedora de recursos as abelhas (Marcucci, 1995), sendo essas variações responsáveis pelas diferenças nas atividades antimicrobianas observadas em diferentes trabalhos (Fernandes Jr. et al., 2006; Gonsalez et al., 2006). Condições de coleta e armazenamento, assim como a resistência do patógeno podem interferir no sucesso dos resultados obtidos.

Com relação à baixa eficiência do óleo de eucalipto sobre o controle de C. musae, devese ressaltar que resultados semelhantes foram relatados em outros estudos. Sousa et al. (2012) determinaram a eficiência de diferentes óleos essenciais sobre C. gloeosporioides, observando que os óleos de babaçu, semente de uva e amêndoa não inibiram o crescimento do respectivo fungo. Segundo Resende et al. (2002), o efeito fungitóxico ou fungistático não é comum a todos os óleos essenciais. Por outro lado, Aguiar et al. (2008) determinaram a eficiência dos óleos de Eucalyptus citriodora e Cymbopogon citratus sobre o fungo $C$. gloeosporioides, encontrando as taxas de inibição na ordem de $100 \%$ para o primeiro e $61 \%$ para o segundo, quando usado nas concentrações de 1000 a $1500 \mu \mathrm{L} / \mathrm{L}$ e $1500 \mu \mathrm{L} / \mathrm{L}$, respectivamente.

Para as avaliações realizadas após 144 e 168 horas da inoculação, os níveis de eficiência do controle do fungo observados para os óleos de tea tree, cravo e palmarosa se mantiveram conforme os observados a partir da terceira avaliação (96h), sugerindo que essas substâncias são eficientes no controle do fungo C. musae (Figura 1). O óleo de eucalipto e própolis mantiveram suas baixas eficiências, chegando a menor dose testada a não diferir da testemunha. Para essas duas substâncias os resultados obtidos não foram semelhantes aos observados para o fungicida, sugerindo que tanto a própolis como o eucalipto não devem ser indicados para o controle de $C$. musae. Observando o gráfico apresentado na Figura 1, nota-se que o eucalipto retomou de forma discreta a relação dose dependente, apresentando a redução do crescimento micelial somente a partir da dose de $75 \mu \mathrm{L} / \mathrm{L}$. Para a própolis essa relação não foi mais observada, sendo o crescimento micelial obtido para a dose de $125 \mu \mathrm{L} / \mathrm{L}$ superior ao obtido para $100 \mu \mathrm{L} / \mathrm{L}$.

A ação fungicida semelhante à observada para os óleos de cravo, palmarosa e tea tree já foi relatada para outros óleos em estudos anteriores. Cruz et al. (2010) determinaram o efeito inibitório de diferentes óleos essenciais no controle do fungo causador da antracnose em frutos de manga, observando que espécies do gênero Citrus são eficazes no controle do fungo, garantindo a qualidade dos frutos no período pós-colheita. Correa-Royero et al. (2010) observaram que as espécies vegetais Chenopodium ambrosioides e Myrcia cucullata apresentam atividade antifúngica sobre Candida krusei.

Outro estudo que comprova a ação fungicida dos óleos essenciais foi realizado por Costa et

Rev. Bras. PI. Med., Campinas, v.17, n.2, p.254-261, 2015. 
naturais danificam a membrana celular, expondo as organelas citoplasmáticas.

Os resultados obtidos para o índice de
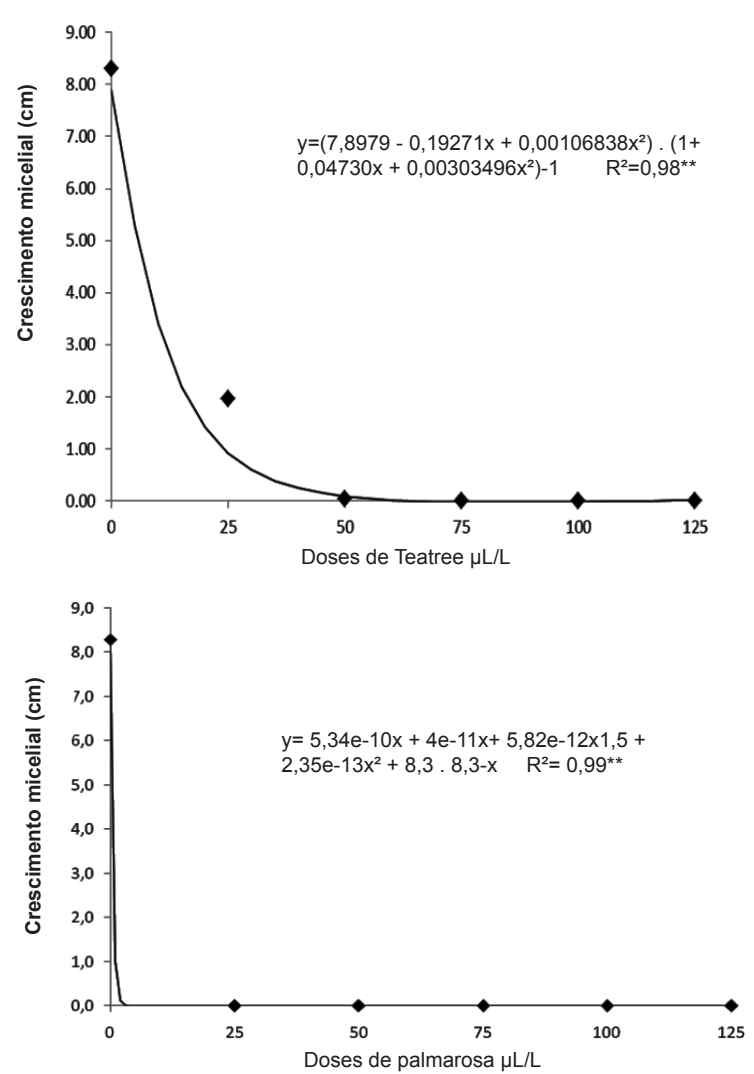

velocidade do crescimento micelial estão em conformidade com os resultados obtidos para o crescimento micelial de C. musae no último dia
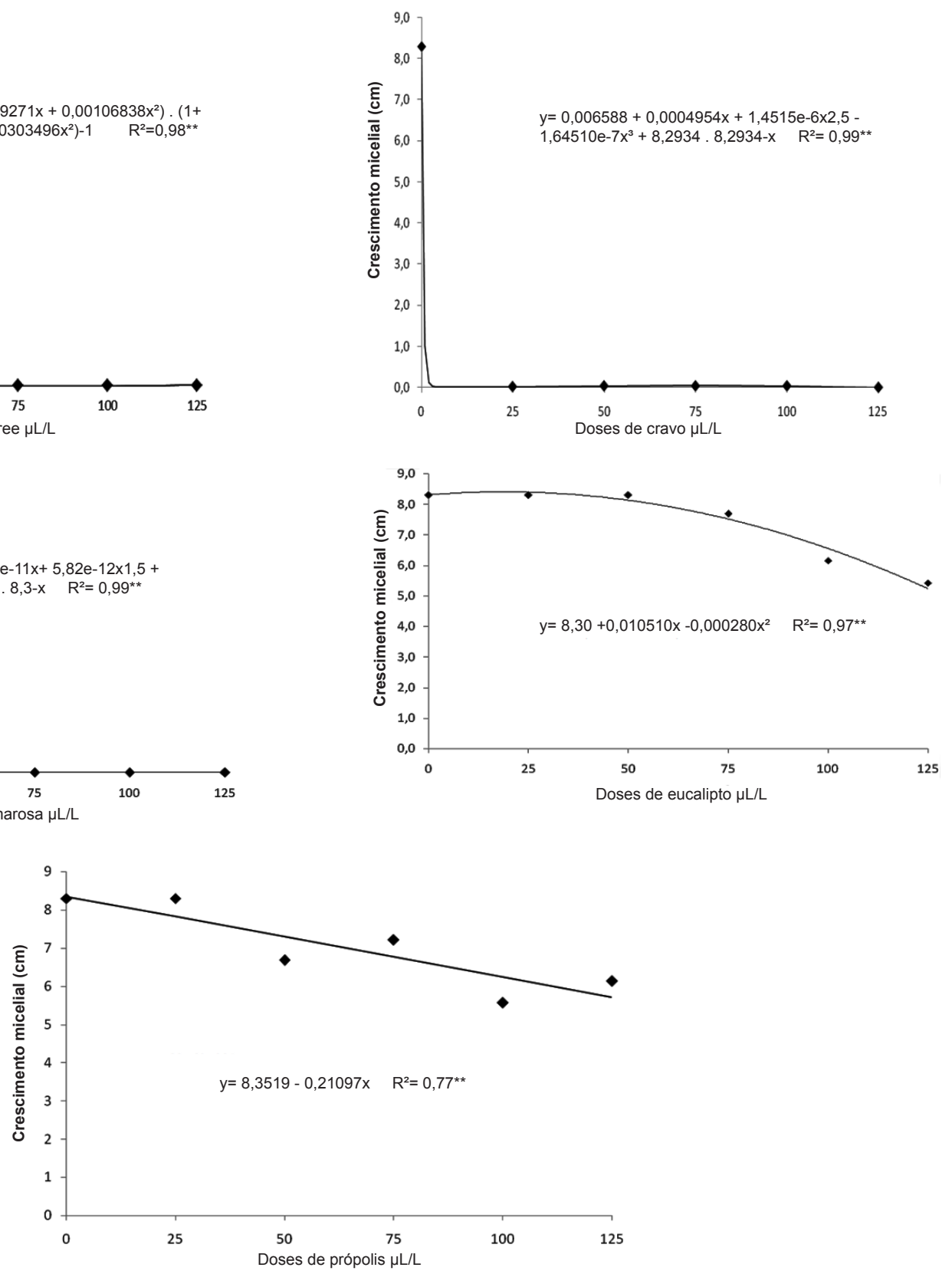

FIGURA 1. Crescimento micelial do fungo C. musae sob efeito de diferentes doses de própolis e óleos essenciais. Cassilândia/MS, 2013.

al. (2011). Esses autores determinaram a ação fungicida do óleo de cravo a $0,15 \%$ sobre os fungos fitopatogênicos Rhizoctonia solani, Fusarium solani, Fusarium oxysporum e Macrophomina phaseolina, observando que apenas o último não foi afetado negativamente por essa substância.

A elevada eficiência observada para o óleo de cravo apresentada em vários estudos se deve principalmente a presença da substância eugenol, que corresponde a $83,6 \%$ dos óleos encontrados nessa estrutura vegetal (Costa et al., 2011). Segundo esses autores, a atividade antifúngica dos óleos essenciais está relacionada a sua hidrofobicidade, que interage com os lipídeos da parede, membrana celular e mitocôndrias, alterando a permeabilidade e causando distúrbios nessas estruturas. Silva et al. (2003) complementaram essas explicações, afirmando que os antifúngicos 
TABELA 2. Índice de velocidade de crescimento micelial (IVCM) do fungo Colletotrichum musae na presença de cinco substâncias alternativas em diferentes concentrações. Cassilândia-MS, 2013.

\begin{tabular}{|c|c|c|c|c|c|}
\hline \multirow{3}{*}{$\begin{array}{l}\text { Doses } \\
(\mu \mathrm{L} / \mathrm{L})\end{array}$} & \multicolumn{5}{|c|}{-IVCM (cm)-- } \\
\hline & & & & & \\
\hline & Própolis & Eucalipto & Teatree & Cravo & Palmarosa \\
\hline Fungicida & $0,00 \mathrm{aE}$ & $0,00 \mathrm{aF}$ & $0,00 \mathrm{aB}$ & $0,00 \mathrm{aB}$ & $0,00 \mathrm{aB}$ \\
\hline Testemunha & $0,44 a A$ & $0,44 a A$ & $0,44 a A$ & $0,44 a A$ & $0,44 a A$ \\
\hline 25 & $0,39 a \mathrm{~B}$ & $0,38 \mathrm{aB}$ & $0,06 \mathrm{bB}$ & $0,00 \mathrm{cB}$ & $0,00 \mathrm{cB}$ \\
\hline 50 & $0,33 b c$ & $0,36 \mathrm{aB}$ & $0,00 \mathrm{cB}$ & $0,00 \mathrm{cB}$ & $0,00 \mathrm{cB}$ \\
\hline 75 & $0,33 a C$ & $0,31 \mathrm{aC}$ & $0,00 \mathrm{bB}$ & $0,00 \mathrm{bB}$ & $0,00 \mathrm{bB}$ \\
\hline 100 & $0,27 \mathrm{aD}$ & $0,23 b D$ & $0,00 \mathrm{cB}$ & $0,00 \mathrm{cB}$ & $0,00 \mathrm{cB}$ \\
\hline 125 & $0,29 \mathrm{aD}$ & $0,17 \mathrm{bE}$ & $0,00 \mathrm{cB}$ & $0,00 \mathrm{cB}$ & $0,00 \mathrm{cB}$ \\
\hline
\end{tabular}

Letras iguais minúsculas nas linhas e maiúsculas nas colunas não diferem entre si pelo teste Scott-Knott a $1 \%$.

de análise para todas as substâncias testadas, ressaltando o potencial dos óleos de palmarosa, cravo e Tea tree no controle desse patógeno (Tabela 2).

Observa-se com base nos resultados apresentados na Tabela 2, que dentre todas as substâncias testadasa própolis foi a que apresentou menor eficiência seguida pelo óleo de eucalipto. Por outro lado, os óleos de palmarosa, cravo e tea tree possuem capacidade inibitória sobre o fungo C. musae, podendo ser usado como uma opção de controle em cultivos orgânicos ou em sistema de manejo integrado, reduzindo assim a aplicação de fungicidas comerciais.

Os estudos sobre óleos essenciais concentram-se principalmente nos óleos de eucalipto, cravo, canela entre outros. Os resultados apresentados no presente estudo ressaltam a eficiência de mais dois óleos disponíveis para o controle de fungos, que são os óleos de tea tree e palmarosa, ambos com propriedades que lhes conferem a condição de possíveis agentes inibitórios sobre o desenvolvimento de fungos fitopatogênicos.

\section{CONCLUSÃO}

Os óleos essenciais de tea tree, cravo e palmarosa possuem capacidade inibitória sobre o fungo Colletotrichum musae, podendo ser usado como uma opção de controle em cultivos orgânicos ou em sistemas de manejo integrado de bananas.

\section{AGRADECIMENTO}

A Pró-reitoria de Pesquisa e Pós-graduação (UEMS), pelo apoio financeiro concedido durante a realização deste trabalho.

\section{REFERÊNCIAS}

AGUIAR, L.G. de; PEREIRA, A.J.; VIVAS, M.; SILVEIRA, S.F. da; MORAIS, W.B.; JESUS JUNIOR, W.C. de; SILVA, D.G. da. Inibição in vitro do crescimento micelial de Colletotrichum gloeosporioides, por óleo essencial de Eucalyptus citriodora e Cymbopogon citratus. In: ENCONTRO LATINO AMERICANO DE INICIAÇÃO CIENTÍFICA, 12., 2008, São José dos Campos, Proceedings... São José dos Campos, São Paulo, 2008. p. 1-3. Disponível em: <http://www. inicepg.univap.br/cd/INIC_2008/anais/arquivosINIC/ INIC0534_04_A.pdf> Acesso em: 08 de jul. 2013.

BAKKALI, F.; AVERBECK, S.; AVERBECK, D.; IDAOMAR, M. Biological effects of essential oils - A review. Food and Chemical Toxicology, v. 46, n. 1, p. 446-475, 2008.

BRASIL. Ministério da Agricultura, Pesca e Abastecimento. Sistema de Agrotóxicos Fitossanitários - AGROFIT. Disponível em:

<http://extranet.agricultura.gov.br/agrofit_cons/ principal_agrofit_cons>. Acesso em: 12 jul. 2013.

COELHO, A.F.S.; DIAS, M.S.C.; RODRIGUES, M.L.M.; LEAL, P.A.M. Controle pós-colheita da antracnose da banana-prata anã tratada com fungicidas e mantida sob refrigeração. Ciência e Agrotecnologia, v. 34, n. 4, p. 1004-1008, 2010.

CORDEIRO, Z.J.M.; MATOS, A.P. Doenças de banana. Informe Agropecuário, Belo Horizonte, v. 26, n. 228, p. 12-16, 2005.

CORDEIRO, Z.J.M.; KIMATI, H. Doenças da bananeira (Musa spp.). In: KIMATI, H.; AMORIM, L.; BERGAMIN FILHO, A.; CAMARGO, L.E.A.; REZENDE, J.A.M. (Eds.) Manual de Fitopatologia: Doenças das plantas cultivadas, 3aEd., São Paulo, Ed. Agronômica Ceres, 1997, p.13-135.

CORREA-ROYERO, J.; TANGARIFE, V.; DURÁN, C.; STASHENKO, E.; MESA-ARANGO, A. In vitro antifungal activity and cytotoxic effect of essential oils and extracts of medicinal and aromatic plants against Candida krusei and Aspergillus fumigatus. Revista Brasileira de Farmacognosia, v. 20, n.1, p. 734-741, 2010.

COSTA, A.R.T.; AMARAL, M.F.Z.J.; MARTINS, P.M.;

Rev. Bras. PI. Med., Campinas, v.17, n.2, p.254-261, 2015. 
PAULA, J.A.M.; FIUZA, T.S.; TRESVENZOL, L.M.F.; PAULA, J.R.; BARA, M.T.F.. Ação do óleo essencial de Syzygiumaromaticum (L.) Merr. \& L.M.Perry sobre as hifas de alguns fungos fitopatogênicos. Revista Brasileira de Plantas Medicinais, v. 13, n. 2, p. 240-245, 2011.

CRUZ, M.J.S.; CLEMENTE, E.; CRUZ, M.E.S.; MORA, F.; COSSARO, L.; PELISSON, N. Efeito dos compostos naturais bioativos na conservação póscolheita de frutos de mangueira cv. Tommy Atkins. Ciência e Agrotecnologia, v. 34, n. 2, p. 428-433, 2010.

FERNANDES, F.F.; DIAS, A.L.T.; RAMOS, C.L.; IKEGAKI, M.; SUQUEIRA, A.M.; FRANCO, M.C. The "in vitro" antifungal activity evaluation of propolis G12 ethanol extract on Cryptococcus neoformans. Revista Instituto Medicina Tropical, v. 49, n.2, p. 93-95, 2007.

FERNANDES JÚNIOR, A.; LOPES, M.M.R.; COLOMBARI, V.; MONTEIRO, A.C.M.; VIEIRA, E.P. Antimicrobial activity of Apis mellifera propolis from three regions of Brazil. Ciência Rural, v.36, n.1, p. 294-297, 2006.

FERREIRA, D. F. Sisvar: a computer statistical analysis system. Ciência e Agrotecnologia, Lavras-MG, v. 35, n. 6, p. 1039-1042, 2011.

GONSALEZ G.Z.; ORSI, R.O.; FERNANDES JÚNIOR, A.; RODRIGUEIS, P.; FUNARI, S.R.C. Antibacterial activity of propolis collected in different regions of Brazil. Journal of Venomous Animals and Toxins including Tropical Diseases, v. 12, n. 2, p. 276-84, 2006.

GOOS, R. D.; TSCHIRSCH, M. Effect of environmental factors on spore germination, spore survival, and growth of Gloeosporium musarum. Mycologia, v. 54, n. 4, p. 353-367, 1962.

LONGHINI, R.; RAKSA, S.M.; OLIVEIRA, A.C.P.; SVIDZINSKI, T.I.E.; FRANCO, SL.L. Obtenção de extratos de própolis sob diferentes condições e avaliação de sua atividade antifúngica. Revista Brasileira Farmacognosia, v. 17, n. 3, p. 388-395, 2007.

MARCUCCI, M. C. Propolis: chemical composition, biological properties and therapeutic activity. Apidologie, v. 26, n. 1, p. 83-99, 1995.

MARINI, D.; MENSCH, R.; FREIBERGER, M.B.; DARTORA, J.; FRANZENER, G.; GARCIA, R.C.; STANGARLIN, J.R. Efeito antifúngico de extratos alcoólicos de própolis sobre patógenos da videira. Arquivos do Instituto Biológico, v.79, n.2, p.305308, 2012.

MELO, R.M.C.; MELO FILHO, P.A.; CÂMARA, M.P.S.; CÂMARA, C.A.G.; SANTOS, R.C. Prospecção de óleos vegetais para controle da ramulose do algodoeiro. In: CONGRESSO BRASILEIRO DO ALGODÃO, 7. 2009, Foz do Iguaçu. Proceedings... Campina Grande: Embrapa Algodão, 2009, p. 1021-1027.

MONZOTE, L.; CUESTA-RUBIO, O.; FERNANDEZ, M.C.; HERNANDEZ, I.M.; FRAGA, J.; PÉREZ,
K.; KERSTENS, M.; MAES, L.; COS,P. In vitro antimicrobial assessment of Cuban propolis extracts. Memórias do Instituto Oswaldo Cruz, v. 107, n. 1, p. 978-984, 2012.

OLIVEIRA, J.A. Efeito do tratamento fungicida em sementes e no controle de tombamento de plântulas de pepino (Cucumis sativus L.) e pimentão (Capsicum annum L.). 1991. 111p. Dissertação (Mestrado - Área de Concentração em Fitossanidade) - Departamento de Fitossanidade, Universidade Federal de Lavras, Lavras, MG.

PEREIRA, A. dos S.; SEIXAS, F.R.M.S.; NETO. F.R. de A. Própolis: 100 anos de pesquisa e suas perspectivas futuras. Química Nova, v. 25, n. 2, p. 321-326, 2002.

PLOETZ, R.C.; THOMAS, J.E.; SLABAUGH, W.R. Diseases of banana and plantain. In: PLOETZ, R.C. (Ed.). Diseases of tropical fruit crops. Florida: University of Florida (UFAS), 2003. p. 73-134.

PROBST, I.S.; SFORCIN, J.M.; RALL, V.L.M.; FERNANDES, A.A.H.; FERNANDES JÚNIOR, A. Antimicrobial activity of propolis and essential oils and synergism between these natural product. The Journal of Venomous Animals and Toxins including Tropical Diseases, v. 17, n. 2, p. 159167, 2011.

RESENDE, M.L.V.; NOJOSA, G.B.A.; CAVALCANTI, L.S.; AGUILAR, M.A.G.; SILVA, L.H.C.P.; PEREZ, J.O.; ANDRADE, G.C.G.; CARVALHO, G.A.; CASTRO, R.M. Induction of resistance in cocoa against Crinipellis perniciosa and Verticillium dahliae by acibenzolar-S-methyl (ASM). Plant Pathology, v.5, n. 1, p. 621-628. 2002.

SALGADO, A.P.S.P.; CARDOSO, M.G.; SOUZA, P.E.; SOUZA, J.A.; ABREU, C.M.P.; PINTO, J.E.B.P. Estudo dos constituintes químicos do óleo essencial das folhas de Eucalyptus e sua atividade fungitóxica. Ciência e Agrotecnologia, v. 27, n.2, p. 17-22, 2003.

SILVA, E.C.A. Preparo do extrato de própolis legal. Mensagem Doce, v. 70, n. 1, p. 2-3, 2003. Disponível em: <www.apacame.org.br/mensagemdoce/70/msg70. htm>Acesso em: 05 Jul. 2013.

SILVA, S.R.S.; DEMUNER, A.J.; BARBOSA, L.C.A.; ANDRADE, N.J.; NASCIMENTO, E.A.; PINHEIRO, A.L. Análise de constituintes químicos e da atividade antimicrobiana do óleo essencial de Melaleuca alternifolia Cheel. Revista Brasileira de Plantas Medicinais, v.6, n.1, p.63-70, 2003.

SOUSA, R.M.S.; SERRA, I.M.R.S.; MELO, T.A. Efeito de óleos essenciais como alternativa no controle de Colletotrichum gloeosporioides, em pimenta. Summa Phytopathologica, v.38, n.1, p.42-47, 2012.

SPONHOLZ, C.; BATISTA, U.G.; ZAMBOLIM, L.; SALOMÃO, L.C.C.; CARDOSO, A.A. Efeito do tratamento hidrotérmico e químico de frutos de banana 'Prata' no controle da antracnose em pós-colheita. Fitopatologia Brasileira, v. 29, n. 5, p. 480-485, 2004.

STANGARLIN, J.R. Uso de extratos e óleos essenciais no controle de doenças de plantas. Fitopatologia Brasileira, v. 32, suplemento, p. 94-6, 2007. 